\title{
JET-CLOUD INTERACTIONS IN NGC 4258: CO OBSERVA- TIONS USING THE NOBEYAMA MILLIMETER ARRAY
}

\author{
R. L. PLANTE ${ }^{1}$, T. HANDA ${ }^{2}$, K. Y. LO ${ }^{1}$ \\ ${ }^{1}$ University of Illinois \\ 103 Astronomy, 1002 W. Green St., Urbana, IL 61801 \\ ${ }^{2}$ Institute of Astronomy, University of Tokyo \\ Mitaka, Tokyo 181
}

\section{INTRODUCTION}

Recent CO studies of spiral galaxy NGC 4258 have revealed a close relationship between the molecular gas and the anomalous arms, recently interpreted as jets (Cecil et al. 1992, Martin et al. 1990). An IRAM single dish study by Krause et al. (1991) showed the $\mathrm{CO}$ to be concentrated around the $\mathrm{H} \alpha$ arms. Interferometric imaging of the inner $1^{\prime}$ by Martin et al. (1990) showed the molecular gas confining the arms; they suggested that the jet had initially plowed a tunnel through the disk gas, producing a free path for subsequent ejections. This data also shows strong evidence for a jet-cloud collision along the NW arm (Plante et al. 1991).

To learn more about the jets, we are motivated to exploit the jet-gas relationship with multi-field imaging of the $\mathrm{CO}$ along the anomalous arms using the Nobeyama Millimeter Array. We present preliminary results from our first $60^{\prime \prime}$ field, centered on the SE $\mathrm{H} \alpha$ arm, $43.5^{\prime \prime}$ from the nucleus. Observations in the two most compact configurations ( $\mathrm{C}$ and $\mathrm{D}$ ) produced a $4^{\prime \prime} \times 6^{\prime \prime}$ beam.

\section{RESULTS}

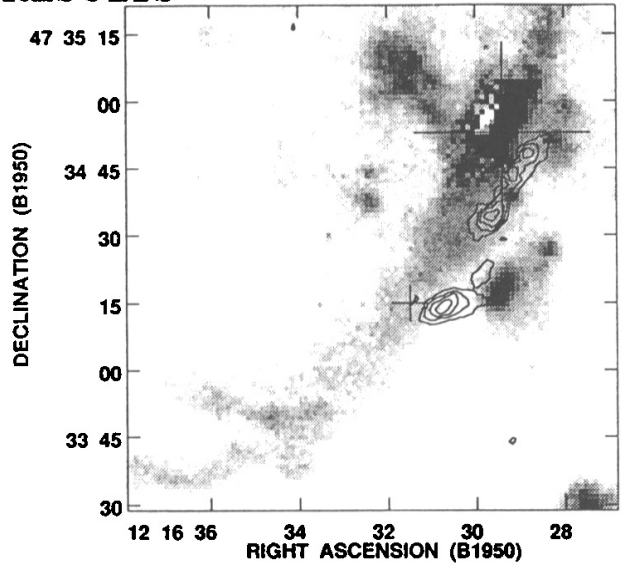

Fig. 1 (above): $\mathrm{CO}$ integrated intensity contours overlayed $\mathrm{H} \alpha$ greyscale. The large cross is the galactic nucleus; the small cross the NMA field center.

Fig. 2 (right): $\mathrm{CO}$ rotation velocities (points), $\mathrm{H} \alpha$ rotation curve (solid line), HI rotation curve (shaded band). Adapted from Cecil et al. 1992.

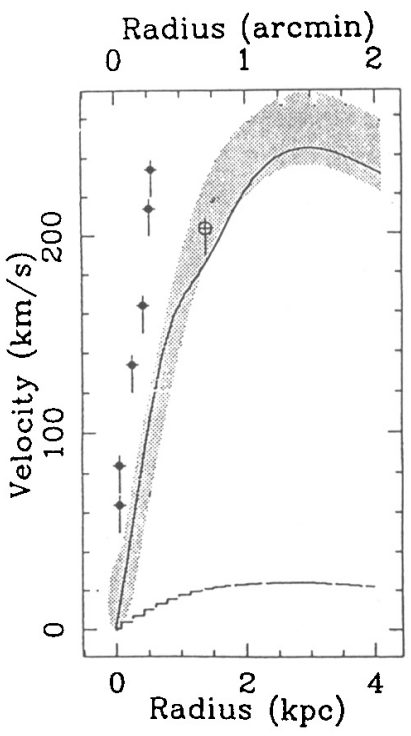


Figure 1 shows the integrated intensity contours of the detected $\mathrm{CO}$ emission between $V_{L S R}=190$ and $440 \mathrm{~km} \mathrm{~s}^{-1}\left(V_{s y s}=464 \mathrm{~km} \mathrm{~s}^{-1}\right.$, Cecil et al. 1992). While some of this emission lies beyond the half-power point of the primary field, the channel maps agree well with those from OVRO observations of the nucleus (see Plante et al. 1991) in both the position and intensity of the emission after taking into account the different synthesized and primary beams. Comparing the $\mathrm{CO}$ integrated intensity with the $\mathrm{H} \alpha$ (greyscale in Fig. 1) shows the CO emission to be concentrated adjacent to and on the downstream side of the jet.

Figure 2 shows that when the velocities of the peaks of the $\mathrm{CO}$ emission are converted to rotational velocities, they appear much higher than the velocities predicted by the bar-forced rotation models for both $\mathrm{HI}$ and $\mathrm{H} \alpha$ (van Albada 1980, Cecil et al. 1992).

\section{POSSIBILITIES}

The spatial and kinematic structure of the $\mathrm{CO}$ does not appear to be a simple reflection of the gravitational potential characterized by the weak bar seen in the observations of $\mathrm{HI}$ and $\mathrm{H} \alpha$. The $\mathrm{CO}$ gas is aligned along the $P A \approx-30^{\circ}$, not along the putative bar which is believed to by oriented at $P A \sim 17^{\circ}$ (van Albada 1980). Furthermore, if we assume this orientation of the bar, we would expect the gas velocities (relative to $V_{\text {sys }}$ ) in the region of the $\mathrm{CO}$ to be less negative-or lower on the graph in Fig. 2-than those expected for circular rotation.

The result illustrated in Fig. 2 may be suggesting an out-of-disk geometry. Again assuming the orientation of the bar as well as an inclination angle $\left(i=72^{\circ}\right.$, van Albada 1980), the velocities of the $\mathrm{CO}$ emission might be explained if the alignment of the $\mathrm{CO}$ gas (and thus the $\mathrm{SE}$ jet) points away from the line of sight about $18^{\circ}$ below the galaxy's disk. However, many convincing arguments have been put forth for the jet axis lying much closer to the plane of the galaxy (van Albada and van der Hulst 1982, Hummel et al. 1989, Cecil et al. 1992).

If we can rule out the out-disk model and if the bar does not explain the high apparent rotation velocities of the $\mathrm{CO}$, then we should consider possible accelleration of the gas by the jet, namely through entrainment or precession. Since the jet lies along the major axis in the region of the $\mathrm{CO}$, entrainment of gas by the jet is not a likely explanation. However, the jet could be precessing through the disk slightly faster than the disk gas. This would allow the jet to sweep up gas and boost its velocity along the line of sight. This model is attractive as it explains simultaneously the spatial and kinematic structures of the $\mathrm{CO}$-including the higher slope of the $\mathrm{CO}$ rotation curve-however, the details about the stability of the jet and the jet walls under precession are as of yet unclear.

\section{REFERENCES}

Cecil, G., Wilson, A.S., \& Tully, R.B. 1992, ApJ, 390, 365

Hummel, E., Krause, M., \& Lesch, H. 1989, $A \mathcal{E} A, \mathbf{2 1 1}, 266$

Krause, M., Cox, P., Garcia-Barreto, J.A., \& Downes, D. 1991, $A \& A$, 233, L1

Martin, P., Roy, J-R., Noreau, L., and Lo, K. Y. 1989, $A p J, \mathbf{3 4 5}, 707$

Plante, R.L., Lo, K.Y., Roy, J-R, Martin, P., \& Noreau, L. 1991, $A p J$, 381, 110

van Albada, G. D. 1980, $A \& B A, 90,123$

van Albada, G. D. \& van der Hulst, J.M. 1982, $A \& A, 115,263$ 